\title{
Increased Fecal Lactate and Decreased Volatile Fatty Acid (VFA), Particularly $n$ - Butyrate Concentrations in Diarrheic Young Calves
}

\author{
Hiroshi SATO ${ }^{1) *}$ \\ ${ }^{1)}$ Department of Large Animal Clinical Sciences, School of Veterinary Medicine, Rakuno Gakuen University, Bunkyodai, Ebetsu, \\ Hokkaido 069-8501, Japan
}

(Received 8 August 2008/Accepted 23 September 2008)

ABSTRACT. Using 24 diarrheic dairy calves under 8 weeks old, multiple fecal samples (4-12) were collected individually during the clinical advancing (max. 10 days) to evaluate the importance of fecal ammonia, lactate and volatile fatty acid (VFA) levels. Removing 3 calves not recovered during the sampling, 21 calves were grouped into under 3 weeks $(<3 \mathrm{wk} ; \mathrm{n}=11)$ and $3-8$ weeks old $(3-8$ wk; $n=10)$. Data were divided into diarrheic and recovered feces with averaging in individuals. Diarrheic feces showed lower VFA and $n$-butyrate, and higher acetate proportions than recovered feces at $<3 \mathrm{wk}$, but not at 3-8 wk. Diarrhea showed higher lactate, and lower ammonia and minor VFA ( $i$-butyrate, valerate), which might reflect insufficiency in gut flora and fermentation.

KEY WORDS: calf diarrhea, colonic fermentation, fecal ammonia, fecal VFA, feces lactate.

Many calves suffer with diarrhea in early life by infectious, nutritional and other factors. The large intestine has an important function in relation to diarrheic pathophysiology. Nutrients that escaped small intestinal digestion are exposed to large bowel fermentation accompanying various intermediates and final products. Comparing with adults, the colon of young mammals is a primary site of microbial colonization because they get most nutrients from liquid milk, not from solid diets. Moreover, gut flora of neonatal mammals is less developed [3] and the immature flora produce large quantities of intermediates such as lactate, succinate and alcohols in calves [8,9]. With advancing age and gut flora, most of these intermediates are converted mainly to volatile fatty acid (VFA) which consist predominant anions in the large bowel [2]. Calf diarrhea has broken out by the upset of delicate balance between calves' gut integrity and gut flora during the postnatal life; however, information on fecal fermentative properties including organic anions is limited in relation to diarrhea. Therefore, the aim of the present study was to elucidate the fecal profiles brought by colonic fermentation within individual calves which experienced both of diarrhea and recovery during the clinical advancing.

Twenty-four diarrheic calves ( 22 male and 2 female; 22 Holstein and 2 Jersey) visited the animal clinic of Rakuno Gakuen University were used. The calves were less than 8 weeks old at fecal collection described latter. They were given approximate $4 \mathrm{~kg}$ of milk replacer daily and were free accessed to water, calf starter and hay. Twelve calves infected with cryptosporidium and two had coccidia; however, the cause of the diarrhea was not fully evaluated in the others. Some calves were given probiotics and medical

\footnotetext{
* Correspondence to: Sato, H., School of Veterinary Medicine, Rakuno Gakuen University, Bunkyodai, Ebetsu, Hokkaido 0698501, Japan.

e-mail : satoh@rakuno.ac.jp
}

charcoal with the milk replacer, but no antibiotics.

In each calf, multiple fecal samples were collected from the rectum during 4-10 days period of clinical advancing. In some calves, fecal samples were taken twice daily or by interval of 2 to 3 days. Sample size in individual calves was 4-12, and total 182 feces were collected from the 24 calves. In 21 calves, both of diarrheic and recovered feces were collected by the successive sampling, but 3 calves showed no recovery during the collection. Fresh feces were extracted with four parts of water immediately after the collection, and $\mathrm{pH}$ was measured. The extract was centrifuged $(2,500$ $\mathrm{g}, 10 \mathrm{~min}$ ), and the supernatant was frozen at $-30^{\circ} \mathrm{C}$ till analyses of ammonia, lactate and VFAs [7].

The data of 3 calves not recovered during fecal collection were excluded. Finally, 165 fecal (86 diarrheic and 79 recovered) samples collected from 21 calves were used for comparison. Data were divided and averaged into 2 categories of diarrheic and recovered feces in each calf, although the diarrheic properties were different among the calves and with clinical advancing. Furthermore, the calves were classified into two groups; i.e., under 3 weeks ( $<3 \mathrm{wk}$; $\mathrm{n}=11)$, and $3-8$ weeks old $(3-8 \mathrm{wk} ; \mathrm{n}=10)$ by the age of incipient diarrhea. At each stage, the difference between diarrheic and recovered feces was statistically evaluated by paired $t$ test.

Comparisons of fecal $\mathrm{pH}$, ammonia, lactate, VFA levels and $n$-butyrate proportions between diarrheic and recovered status in each calf were shown in Fig. 1. Large individual differences were observed in ammonia, VFA and particularly in lactate levels. Statistical comparisons of fecal properties between the diarrhea and recovery at each stage are given in Table 1.

Careful considerations should be given in the study of neonatal calf diarrhea. Since the fecal profiles had large individual difference during the neonatal period irrespective of the same feeding of milk $[8,9]$, a comparative study of 
fecal profiles among the calves has serious difficulties in explanation. Therefore, the present data were compared between normal and diarrheic feces in the individuals, and not between the normal and diarrhea of different calves. Secondary, an averaging all the diarrheic data which include various fecal properties, i.e., pasty, watery or runny samples, might be problem. However in the present study, the diarrheic data in each calf were averaged for describing the outline of calf diarrhea. Thirdly, the present data were divided into 2 stages of $<3 \mathrm{wk}$ and 3-8 wk old, because neonatal calves within few weeks after birth had an immature flora and marked fluctuations in gut fermentation compared with the calves of the following stage despite the same feedings [9]. Even so, these fluctuations in the early life were far from the metabolic and hormonal changes caused by the development of solid feed intake [1].

In diarrheic feces, lactate concentration was significantly higher and ammonia was significantly lower than those in recovered feces by paired $t$-test; a declined lactate in recov- ery from diarrhea was evident in 17 of 21 calves (Fig. 1). Fecal $\mathrm{pH}$ in diarrhea was lower than that in recovered status at $3-8 \mathrm{wk}$, but not at the early stage. Lactate is an intermediate product in the global fermentation process, and the acid is finally metabolized to VFA by entire gut flora [2]. The increased fecal lactate in diarrhea agrees well with the report in calves of naturally occurring diarrhea [15]. The increased fecal lactate seemed to be a reflection of anaerobic fermentation of milk lactose which escaped digestion in the small intestine. According to the dissociation constant (pK), lactate is a strong acid than VFA [4]; however, fecal acidity at early stage $(<3 \mathrm{wk})$ showed less dependence on lactate concentration. Thus, the fecal acidity in postnatal calves might be dominated by not only strong acids including lactate but also luminal electrolytes and others [6]. Lactic fermentation might help gut colonization against pathogens in neonates; however severe colitis was observed in human patients who had extremely higher lactate in the colon [13].

Diarrhea brought a significant decrease in fecal VFA,
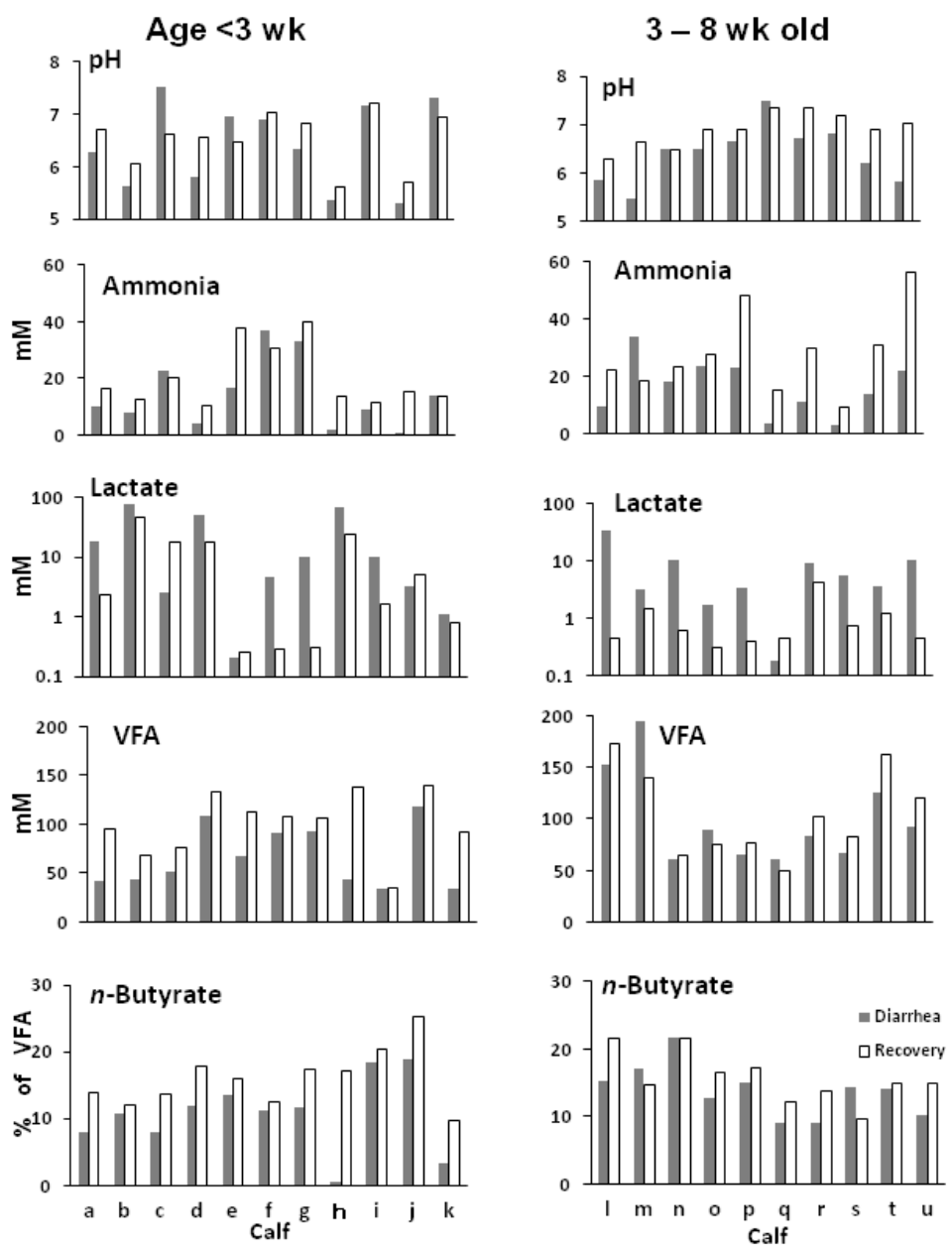

Fig. 1. Comparison of fecal $\mathrm{pH}$, ammonia, lactate and volatile fatty acid (VFA) levels between diarrheic and recovered status in individula calves. a-u, individual calves. 
Table 1. Comparison of ammonia, lactate and volatile fatty acid (VFA) concentrations between in diarrheic and recovered feces in young calves

\begin{tabular}{|c|c|c|c|c|c|c|c|c|c|c|}
\hline \multirow{2}{*}{$\begin{array}{l}\text { Age } \\
\text { (week) }\end{array}$} & \multirow[t]{2}{*}{ Feces } & & \multirow[t]{2}{*}{$\mathrm{pH}$} & \multirow{2}{*}{$\begin{array}{c}\text { Ammonia } \\
(\mathrm{mM})\end{array}$} & \multirow{2}{*}{$\begin{array}{l}\text { Lactate } \\
(\mathrm{mM})\end{array}$} & \multirow{2}{*}{$\begin{array}{l}\text { VFA } \\
(\mathrm{mM})\end{array}$} & Acetate & Propionate & $n$-Butyrate & $i-\mathrm{B}+\mathrm{V}^{\mathrm{a}}$ \\
\hline & & & & & & & \multicolumn{4}{|c|}{ (Molar \%) } \\
\hline \multirow{4}{*}{$\begin{array}{c}<3 \\
(\mathrm{n}=11)\end{array}$} & Diarrheic & Mean & 6.41 & $14.6^{*}$ & $22.8^{*}$ & $67.0^{* *}$ & $62.1 * *$ & 22.8 & $10.5 * *$ & $4.6^{*}$ \\
\hline & & $\mathrm{SD}$ & 0.82 & 12.1 & 29.4 & 31.3 & 13.1 & 8.4 & 5.6 & 3.9 \\
\hline & Recovered & Mean & 6.52 & 20.1 & 10.6 & 100.6 & 52.8 & 24.0 & 16.0 & 7.1 \\
\hline & & $\mathrm{SD}$ & 0.52 & 10.8 & 14.4 & 32.0 & 8.3 & 3.6 & 4.4 & 4.5 \\
\hline \multirow{4}{*}{$\begin{array}{c}3-8 \\
(n=10)\end{array}$} & Diarrheic & Mean & $6.41 * *$ & $16.4 *$ & $8.4^{*}$ & 99.2 & 50.6 & $29.4 * *$ & 13.9 & $6.1 *$ \\
\hline & & $\mathrm{SD}$ & 0.58 & 9.8 & 10.1 & 44.8 & 12.3 & 7.9 & 3.9 & 3.8 \\
\hline & Recovered & Mean & 6.91 & 27.9 & 1.0 & 105.0 & 48.7 & 24.9 & 15.7 & 10.7 \\
\hline & & SD & 0.35 & 14.4 & 1.2 & 42.4 & 12.2 & 4.6 & 3.8 & 6.5 \\
\hline
\end{tabular}

Diarrheic and recovered samples were obtained from the same individuals within 10 days of clinical advancing, and plural data within each individual were averaged.

Eleven $(<3$ weeks old) and ten (3-8 weeks) sets of diarrheic and recovered fecal samples from the individuals were statistically evaluated by paired $t$-test.

*,**: Significance compared to recovered feces $\left(* \mathrm{P}<0.05,{ }^{* *} \mathrm{P}<0.01\right)$. ${ }^{\text {a: }}$ Sum of $i$-butyrate and valerate.

particularly $n$-butyrate with a compensatory increase in acetate proportion at the early stage, but these VFAs showed no significant difference at 3-8 wk. Importance of VFA must be noticed in maintaining gut integrity and its function. It is known that luminal production of VFA has important role in maintaining the morphologic and functional integrity of the gut $[5,11,14]$, and in electrolyte movement in the intestine $[10,11]$. Moreover, $n$-butyrate is preferentially used for energy by the colon [12], and $n$-butyrate deficiency results in colitis and malignization of colonocytes in humans [10]. Thus, $n$-butyrate production might be essential in the colon particularly in an early life of calves.

Recovered feces had higher proportion of the sum of fecal $i$-butyrate, $i$-valerate and $n$-valerate (minor VFA), and higher fecal ammonia (Table 1). Increase of the minor VFA with recovery is worth noting. These acids are produced by fermentation of amino acids that originated from proteolysis in the gut. Increased production of these VFA, including branched acids, might be reflection of improved fermentation by plentiful gut microbes in the recovered colon. Indeed in a study of fecal VFA profile of human infants, acetate production easily reached to adult level at the first with the following of propionate and $n$-butyrate, however the production of the minor VFA lagged far behind the three major acids [3]. Increased minor VFA in recovered status might be a reflection of improved gut flora and its fermentation.

In conclusion, a higher lactate and lower ammonia were confirmed in diarrheic feces of young calves, and fecal VFA, particularly $n$-butyrate decreased in early postnatal diarrhea.

\section{REFERENCES}

1. Baldwin, R.L.,VI., McLeod, K.R., Klotz, J.L. and Heitmann, R.N. 2004. Rumen development, intestinal growth and hepatic metabolism in the pre- and postweaning ruminant. J. Dairy Sci. 87: E55-E65.

2. Macfarlane, S. and Macfarlane, G.T. 2003. Regulation of short-chain fatty acid production. Proc. Nutr. Soc. 62: 67-72.
3. Mackie, R.I., Sghir, A. and Gaskins, H.R. 1999. Developmental microbial ecology of the neonatal gastrointestinal tract. Am. J. Clin. Nutr. 69: 1035S-1045S.

4. Partanen, K.H. and Mroz, Z. 1999. Organic acids for performance enhancement in pig diets. Nutr. Res. Rev. 12: 117-145.

5. Sakata, T. 1987. Stimulatory effect of short-chain fatty acids on epithelial cell proliferation in the rat intestine: a possible explanation for trophic effects of fermentable fibre, gut microbes and luminal trophic factors. Br. J. Nutr. 58: 95-103.

6. Sato, H. and Koiwa, M. 2008. Fecal D- and L- lactate, succinate and volatile fatty acid levels, and relationships with fecal acidity and diarrhea in neonatal calves. Anim. Sci. J. 79: 187192.

7. Sato. H. and Nakajima, J. 2005. Fecal ammonia, urea, volatile fatty acid and lactate levels in dairy cows and their pathophysiological significance during diarrhea. Anim. Sci. J. 76: 595599.

8. Sato, H. and Shiogama, Y. 2009. Fecal excretion of alcohols and organic anions in neonatal dairy calves. Anim. Sci. J. 80: (in press).

9. Shimomura, Y. and Sato, H. 2006. Fecal D- and L-lactate, succinate, and volatile fatty acid levels in young dairy calves. $J$. Vet. Med. Sci. 68: 973-977.

10. Soergel, K.H. 1994. Colonic fermentation: metabolic and clinical implications. Clin. Inv. 72: 742-748.

11. Topping, D.L. and Clifton, P.M. 2001. Short-chain fatty acids and human colonic function: Roles of resistant starch and nonstarch polysaccharides. Physiol. Rev. 81: 1031-1064.

12. Velazquez, O.C., Seto, R.W. and Rombeau, J.L. 1996. The scientific rationale and clinical application of short-chain fatty acids and medium-chain triacylglycerols. Proc. Nutr. Soc. 55: 49-78.

13. Vernia, P., Caprilli, R., Latella, G., Barbetti, F., Magliocca, F.M. and Cittadini, M. 1988. Fecal lactate and ulcerative colitis. Gastroenterology 95: 1564-1568.

14. Williams, B.A., Verstegen, M.W.A. and Tamminga, S. 2001. Fermentation in the large intestine of single-stomached animals and its relationship to animal health. Nutr. Res. Rev. 14: 207227.

15. Youanes, Y.D. and Herdt, T.H. 1987. Changes in small intestinal morphology and flora associated with decreased energy digestibility in calves with naturally occurring diarrhea. Am. J. Vet. Res. 48: 719-725. 